\title{
Verification and comparison of MIT-BIH arrhythmia database based on number of beats
}

\author{
Akram Jaddoa Khalaf, Samir Jasim Mohammed \\ Department of Electrical Engineering, Faculty of Engineering, University of Babylon, Iraq
}

\begin{tabular}{|c|c|}
\hline Article Info & ABSTRACT \\
\hline Article history: & \multirow{11}{*}{$\begin{array}{l}\text { The ECG signal processing methods are tested and evaluated based on many } \\
\text { databases. The most ECG database used for many researchers is the MIT- } \\
\text { BIH arrhythmia database. The QRS-detection algorithms are essential for } \\
\text { ECG analyses to detect the beats for the ECG signal. There is no standard } \\
\text { number of beats for this database that are used from numerous researches. } \\
\text { Different beat numbers are calculated for the researchers depending on the } \\
\text { difference in understanding the annotation file. In this paper, the beat } \\
\text { numbers for existing methods are studied and compared to find the correct } \\
\text { beat number that should be used. We propose a simple function to } \\
\text { standardize the beats number for any ECG PhysioNet database to improve } \\
\text { the waveform database toolbox (WFDB) for the MATLAB program. This } \\
\text { function is based on the annotation's description from the databases and can } \\
\text { be added to the Toolbox. The function is removed the non-beats annotation } \\
\text { without any errors. The results show a high percentage of } 71 \% \text { from the } \\
\text { reviewed methods used an incorrect number of beats for this database. }\end{array}$} \\
\hline Received Nov 24, 2020 & \\
\hline Mar 30, 2021 & \\
\hline Accepted Apr 9, 2 & \\
\hline Keywords: & \\
\hline ECG & \\
\hline Heartbeat & \\
\hline MATLAB & \\
\hline MIT-BIH arrhythmia database & \\
\hline QRS-detection & \\
\hline & \\
\hline
\end{tabular}

This is an open access article under the CC BY-SA license.

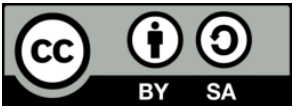

\section{Corresponding Author:}

Akram Jaddoa Khalaf

Department of Electrical Engineering

University of Babylon

Hilla, Babylon, Iraq

Email: eng.akram@uobabylon.edu.iq

\section{INTRODUCTION}

The electrocardiogram (ECG) was generally used for the observation of cardiac physiology as a cost-effective and non-invasive process. For the cardiologist to diagnose cardiac diseases, the ECG signal shows heart functionality. The ECG field is developed significantly, considering the most common death is generally from cardiovascular diseases [1]. Many applications are based on the ECG signal, such as measuring the heart-rate, biometric-identification, movement-recognize, and diagnosing-abnormality [2].

Generally, the first ECG standard material available for testing and performance evaluation is the MIT-BIH arrhythmia database [3]. It played together with the American Heart Association (AHA) database an interesting role in stimulating manufacturers of arrhythmia analyzers to compete on the basis of objectively measurable performance. The value of common databases for basic research and medical device development and evaluation is attributed to the MIT-BIH arrhythmia database. The MIT-BIH has comprised variable ECG signals with a variable: noise, artifacts, beat types, and wave shapes. A 48-records with two channels for each ECG-signal and an annotation file are included. These signals are recording from 25 men and 22 women for a half-hour period at 360 samples per second. The database has been annotated with 112,647 annotations, and these annotations have been verified [3]. It has been classified into two main annotation categories: the beats and the non-beats. The beat annotations for the MIT-BIH arrhythmia database consist of 15 subtypes, and the non-beats annotations consist of 24 subtypes, as shown in Table 1. 
The beat type consists of 14 classified beats types and one unclassified beats type. The beats annotations are occurring for any type of QRS waves in ECG-signal. Therefore, this database is widely used for testing, performance evaluating, and learning for QRS-detection methods. In general, the databases are used to evaluate any new algorithm's performance before implementing it in devices for many applications. So, any errors for this evaluation will cause an error in the device decision. In biomedical applications like QRSdetection, which is substantial for many ECG monitoring devices, the detecting errors for these devices may affect doctor's diagnosis and treatment depending on these devices. So, verifying the database for these applications will improve the doctor's decision.

Table 1. The description of beat and non-beat annotations for MIT-BIH arrhythmia database

\begin{tabular}{|c|c|c|c|}
\hline \multicolumn{2}{|c|}{ Beat annotations } & \multicolumn{2}{|c|}{ Non-beat annotations } \\
\hline Code & Description & Code & Description \\
\hline $\mathrm{N}$ & Normal beat & {[} & Start of ventricular flutter/fibrillation \\
\hline $\mathrm{L}$ & Left bundle branch block beat & ! & Ventricular flutter wave \\
\hline $\mathrm{R}$ & Right bundle branch block beat & ] & End of ventricular flutter/fibrillation \\
\hline A & Atrial premature beat & $\mathrm{x}$ & Non-conducted P-wave (blocked APC) \\
\hline a & Aberrated atrial premature beat & $(\mathrm{N}$ & Normal sinus rhythm \\
\hline $\mathrm{J}$ & Nodal (junctional) premature beat & $(\mathrm{P}$ & Paced rhythm \\
\hline $\mathrm{S}$ & Supraventricular premature or ectopic beat (atrial or nodal) & (B & Ventricular bigeminy \\
\hline $\mathrm{V}$ & Premature ventricular contraction & (VT & Ventricular tachycardia \\
\hline $\mathrm{F}$ & A fusion of ventricular and normal beat & $(\mathrm{T}$ & Ventricular trigeminy \\
\hline e & Atrial escape beat & (SVTA & Supraventricular tachyarrhythmia \\
\hline $\mathrm{j}$ & Nodal (junctional) escape beat & (IVR & Idioventricular rhythm \\
\hline $\mathrm{E}$ & Ventricular escape beat & (NOD & Nodal (A-V junctional) rhythm \\
\hline / & Paced beat & (AFIB & Atrial fibrillation \\
\hline $\mathrm{f}$ & A fusion of paced and normal beat & (AFL & Atrial flutter \\
\hline \multirow[t]{10}{*}{ Q } & Unclassifiable beat & (VFL & Ventricular flutter \\
\hline & & $(\mathrm{AB}$ & Atrial bigeminy \\
\hline & & (PREX & Pre-excitation (WPW) \\
\hline & & (BII & $2^{\circ}$ heart block \\
\hline & & (SBR & Sinus bradycardia \\
\hline & & $\mid$ & Isolated QRS-like artifact \\
\hline & & $\sim$ & Change in signal quality \\
\hline & & ":TS & Tape slippage \\
\hline & & ":PSE & Pause \\
\hline & & ":MISSB & Missed beat \\
\hline
\end{tabular}

Until now, more than two thousand works cited the MIT-BIH arrhythmia database. It is unique in terms of arrhythmia classification since it offers five arrhythmia standards groups [2]. The QRS detection methods are essential for most of the cited works, including arrhythmia detection, classification, and diagnosing applications. Depending on this database, many QRS detection algorithms have been developed, tested, and evaluated. The QRS detection algorithms are based on the beats annotations in the database signals for testing and evaluation. These beats are used as learning data for the methods depending on the learning technique.

Many researchers used MATLAB for algorithm implementation based on the waveform database (WFDB) Toolbox [4]. This Toolbox consists of the functions that are used for reading, writing, and signals processing the files of PhysioNet databases. The MIT-BIH arrhythmia is one of the PhysioNet databases which contains data and annotations files. The WFDB is used to extract the ECG-signals and these annotations from the MIT-BIH arrhythmia database for all records. It can extract one type of beats or nonbeats annotations or extract all annotations without any filter. So, it is not easy to extract all beat annotations only, which is leads to errors from reading the non-beat annotations. When reviewing the existing methods that used the MIT-BIH arrhythmia database, we noted that not all these methods are considered the same number of beats for the same database records. Also, this difference affects even slightly the evaluation results used to compare the performance of the methods.

This work will study the reasons for reading different numbers of beats and methods comparison with correction and verification. Furthermore, a new function is designed to extract the correct beats and remove the non-beats annotations from the original database files based on WFDB Toolbox for MATLAB.

In section 2, the MIT-BIH arrhythmia database and its annotation types in detail are described. Section 3 present the proposed function that extracts the correct beat from the annotation files. Then, section 4 demonstrates the results and discussion for revising the existing methods with a comparison based on each method's beat number. Finally, in section 5, the conclusion is summarized. 


\section{MIT-BIH ARRHYTHMIA DATABASE}

The MIT-BIH arrhythmia database is one of the most substantial ECG databases. Contrasting database signals, noise, and artifacts make it suitable for testing and evaluation. Moreover, the verified annotations files that contain the beats and non-beats types, as shown in Table 2 and Table 3. These tables show the MIT-BIH arrhythmia database annotations for each record based on the PhysioNet annotations descriptions for beats and non-beats annotations. There are more than these annotation types, which are shown in other databases.

Table 2. MIT-BIH arrhythmia database beat annotations

\begin{tabular}{|c|c|c|c|c|c|c|c|c|c|c|c|c|c|c|c|c|c|}
\hline $\begin{array}{l}\text { Record } \\
\text { No. }\end{array}$ & $\begin{array}{c}\text { Total } \\
\text { Anno-tations }\end{array}$ & $\mathrm{N}$ & $\mathrm{L}$ & $\mathrm{R}$ & A & $\mathrm{a}$ & $\mathrm{J}$ & $\mathrm{S}$ & V & $\mathrm{F}$ & e & $\mathrm{j}$ & E & I & $\mathrm{f}$ & $\mathrm{Q}$ & $\begin{array}{l}\text { Total } \\
\text { Beats }\end{array}$ \\
\hline 100 & 2274 & 2239 & & & 33 & & & & 1 & & & & & & & & 2273 \\
\hline 101 & 1874 & 1860 & & & 3 & & & & & & & & & & & 2 & 1865 \\
\hline 103 & 2091 & 2082 & & & 2 & & & & & & & & & & & & 2084 \\
\hline 104 & 2311 & 163 & & & & & & & 2 & & & & & 1380 & 666 & 18 & 2229 \\
\hline 105 & 2691 & 2526 & & & & & & & 41 & & & & & & & 5 & 2572 \\
\hline 108 & 1824 & 1739 & & & 4 & & & & 17 & 2 & & 1 & & & & & 1763 \\
\hline 109 & 2535 & & 2492 & & & & & & 38 & 2 & & & & & & & 2532 \\
\hline 111 & 2133 & & 2123 & & & & & & 1 & & & & & & & & 2124 \\
\hline 112 & 2550 & 2537 & & & 2 & & & & & & & & & & & & 2539 \\
\hline 113 & 1796 & 1789 & & & & 6 & & & & & & & & & & & 1795 \\
\hline 118 & 2301 & & & 2166 & 96 & & & & 16 & & & & & & & & 2278 \\
\hline 119 & 2094 & 1543 & & & & & & & 444 & & & & & & & & 1987 \\
\hline 121 & 1876 & 1861 & & & 1 & & & & 1 & & & & & & & & 1863 \\
\hline 122 & 2479 & 2476 & & & & & & & & & & & & & & & 2476 \\
\hline 123 & 1519 & 1515 & & & & & & & 3 & & & & & & & & 1518 \\
\hline 124 & 1634 & & & 1531 & 2 & & 29 & & 47 & 5 & & 5 & & & & & 1619 \\
\hline 200 & 2792 & 1743 & & & 30 & & & & 826 & 2 & & & & & & & 2601 \\
\hline 201 & 2039 & 1625 & & & 30 & 97 & 1 & & 198 & 2 & & 10 & & & & & 1963 \\
\hline 202 & 2146 & 2061 & & & 36 & 19 & & & 19 & 1 & & & & & & & 2136 \\
\hline 203 & 3108 & 2529 & & & & 2 & & & 444 & 1 & & & & & & 4 & 2980 \\
\hline 205 & 2672 & 2571 & & & 3 & & & & 71 & 11 & & & & & & & 2656 \\
\hline 217 & 2280 & 244 & & & & & & & 162 & & & & & 1542 & 260 & & 2208 \\
\hline 219 & 2312 & 2082 & & & 7 & & & & 64 & 1 & & & & & & & 2154 \\
\hline 220 & 2069 & 1954 & & & 94 & & & & & & & & & & & & 2048 \\
\hline 221 & 2462 & 2031 & & & & & & & 396 & & & & & & & & 2427 \\
\hline 222 & 2634 & 2062 & & & 208 & & 1 & & & & & 212 & & & & & 2483 \\
\hline 223 & 2643 & 2029 & & & 72 & 1 & & & 473 & 14 & 16 & & & & & & 2605 \\
\hline 228 & 2141 & 1688 & & & 3 & & & & 362 & & & & & & & & 2053 \\
\hline 230 & 2466 & 2255 & & & & & & & 1 & & & & & & & & 2256 \\
\hline 231 & 2011 & 314 & & 1254 & 1 & & & & 2 & & & & & & & & 1571 \\
\hline 232 & 1816 & & & 397 & 1382 & & & & & & & 1 & & & & & 1780 \\
\hline 233 & 3152 & 2230 & & & 7 & & & & 831 & 11 & & & & & & & 3079 \\
\hline 234 & 2764 & 2700 & & & & & 50 & & 3 & & & & & & & & 2753 \\
\hline Total & 112647 & 75052 & 8075 & 7259 & 2546 & 150 & 83 & 2 & 7130 & 803 & 16 & 229 & 106 & 7028 & 982 & 33 & 109,494 \\
\hline
\end{tabular}

Each beat's annotation is a QRS-complex with different types as normal-beat or other beats. On the other hand, The Non-beat annotations are ventricular flutter wave, start/end of ventricular flutter, and starting for many types of rhythm like (sinus, paced, ventricular, supraventricular, atrial fibrillation, atrial flutter, and heart block). These are annotated ECG signal to show at this point one of the rhythms are starting. So, it is not a beats (QRS) annotation. The ventricular flutter (record 207) is excepted for many QRS-detection methods because it is defined on the ECG by a sinusoidal wave without a clear showing of the QRS-complex 
wave and T wave. The QRS detection methods based on the MIT-BIH arrhythmia database use the beat annotation only because the non-beats annotations are not shown QRS waves for testing, evaluation, and learning.

The number of beats annotations are shown in Table 2 with (109,494 Beats) for all 48 records. This number should be a standard number of beats depending on the original database annotation details and the PhysioNet annotations descriptions for beats and non-beats types. Also, the QRS detection methods are excluded from the 472 ventricular flutter waves from record no. 207, because these waves are considered as non-beat annotations based on the annotation's description of PhysioNet as shown in Table 3.

Table 3. MIT-BIH arrhythmia database non-beat annotations

\begin{tabular}{|c|c|c|c|c|c|c|c|c|c|c|c|c|c|c|c|c|c|c|c|c|c|c|c|c|c|c|}
\hline $\begin{array}{c}\text { Record } \\
\text { No. }\end{array}$ & $\begin{array}{l}\text { Total } \\
\text { Anno- } \\
\text { tations }\end{array}$ & 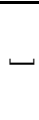 & - & $\neg$ & $x$ & 乙 & $\theta$ & $\vartheta$ & $\stackrel{5}{2}$ & $\bullet$ & $\underset{⿱ ㇒}{\leftrightarrows}$ & $\stackrel{q}{己}$ & 己 & $\stackrel{\oplus}{\longleftarrow}$ & 崖 & $\stackrel{+}{2}$ & $\stackrel{2}{\longleftarrow}$ & 宏 & $\ddot{\vartheta}$ & $\frac{\tilde{n}}{\sqrt[n]{n}}$ & - & 2 & $\stackrel{\mathscr{L}}{=}$ & $\begin{array}{l}\stackrel{1}{\mathscr{2}} \\
\stackrel{0}{=}\end{array}$ & $\sum_{\infty}^{\infty}$ & Total Non-Beats \\
\hline 101 & 1874 & & & & & 1 & & & & & & & & & & & & & & & 4 & 4 & & & & 9 \\
\hline 102 & 2192 & & & & & 2 & 3 & & & & & & & & & & & & & & & & & & & 5 \\
\hline 103 & 2091 & & & & & 1 & & & & & & & & & & & & & & & & 6 & & & & 7 \\
\hline 106 & 2098 & & & & & 21 & & 18 & 1 & 1 & & & & & & & & & & & & 30 & & & & 71 \\
\hline 107 & 2140 & & & & & & 1 & & & & & & & & & & & & & & & 2 & & & & 3 \\
\hline 108 & 1824 & & & & 11 & 1 & & & & & & & & & & & & & & & 8 & 41 & & & & 61 \\
\hline 109 & 2535 & & & & & 1 & & & & & & & & & & & & & & & & 2 & & & & 3 \\
\hline 111 & 2133 & & & & & 1 & & & & & & & & & & & & & & & & 8 & & & & 9 \\
\hline 112 & 2550 & & & & & 1 & & & & & & & & & & & & & & & & 10 & & & & 11 \\
\hline 116 & 2421 & & & & & 1 & & & & & & & & & & & & & & & & 8 & & & & 9 \\
\hline 117 & 1539 & & & & & 1 & & & & & & & & & & & & & & & & 3 & & & & 4 \\
\hline 118 & 2301 & & & & 10 & 1 & & & & & & & & & & & & & & & & 12 & & & & 23 \\
\hline 119 & 2094 & & & & & 49 & & 37 & & 17 & & & & & & & & & & & & 4 & & & & 107 \\
\hline 121 & 1876 & & & & & 1 & & & & & & & & & & & & & & & & 12 & & & & 13 \\
\hline 122 & 2479 & & & & & 1 & & & & & & & & & & & & & & & 2 & & & & & 3 \\
\hline 123 & 1519 & & & & & 1 & & & & & & & & & & & & & & & & & & & & 1 \\
\hline 124 & 1634 & & & & & 6 & & & & 2 & & 3 & 2 & & & & & & & & & 2 & & & & 15 \\
\hline 200 & 2792 & & & & & 70 & & 71 & 7 & & & & & & & & & & & & & 43 & & & & 191 \\
\hline 201 & 2039 & & & & 37 & 16 & & & & 12 & 1 & & 3 & 3 & & & & & & & & 4 & & & & 76 \\
\hline 202 & 2146 & & & & & 3 & & & & & & & & 4 & 1 & & & & & & 2 & & & & & 10 \\
\hline 203 & 3108 & & & & & & & & 21 & 1 & & & & 21 & 2 & & & & & & 26 & 57 & & & & 128 \\
\hline 215 & 3400 & & & & & 3 & & & 2 & & & & & & & & & & & & & 30 & 2 & & & 37 \\
\hline 217 & 2280 & & & & & & 33 & 9 & 1 & & & & & 24 & & & & & & & 1 & 4 & & & & 72 \\
\hline 219 & 2312 & & & & 133 & 8 & & 2 & & 1 & & & & 10 & & & & & & & & & & 3 & 1 & 158 \\
\hline 220 & 2069 & & & & & 9 & & & & & 8 & & & & & & & & & & & 4 & & & & 21 \\
\hline 221 & 2462 & & & & & & & 1 & 2 & 8 & & & & 12 & & & & & & & & 12 & & & & 35 \\
\hline 222 & 2634 & & & & & 32 & & & & & 4 & & 31 & 24 & 42 & & 3 & & & & & 15 & & & & 151 \\
\hline 223 & 2643 & & & & & 11 & & 7 & 7 & 3 & & & & & & & & & & & & 10 & & & & 38 \\
\hline 228 & 2141 & & & & & 21 & & 20 & & & & & & & & & & & & & 24 & 20 & 3 & & & 88 \\
\hline 230 & 2466 & & & & & 104 & & & & & & & & & & & & 103 & & & 1 & 2 & & & & 210 \\
\hline 231 & 2011 & & & & 2 & 6 & & & & & & & & & & & & & 5 & & & & & & 427 & 440 \\
\hline 232 & 1816 & & & & & & & & & & & & & & & & & & & 1 & & 35 & & & & 36 \\
\hline 233 & 3152 & & & & & 36 & & 28 & 6 & 1 & & & & & & & & & & & 2 & & & & & 73 \\
\hline 234 & 2764 & & & & & 2 & & & & & 1 & & & & & & & & & & & 8 & & & & 11 \\
\hline Total & 112647 & 6 & 472 & 6 & 193 & 530 & 60 & 221 & 61 & 83 & 26 & 4 & 36 & 107 & 45 & 6 & 3 & 103 & 5 & 1 & 132 & 616 & 6 & 3 & 428 & 3153 \\
\hline
\end{tabular}

\section{HEARTBEATS FILTER FUNCTION}

In this paper, a MATLAB function is designed to filter the annotations file for any PhysioNet databases included the MIT-BIH arrhythmia. The function removes the non-beat annotation shown in Table 3 so, the annotations file will contain the beat annotation only shown in Table 2. On the other hand, the existing 
MATLAB-WFDB function (rdann) reading the annotations file can read all annotations or one annotation. So, rdann cannot filter the annotation by beats or non-beats type; for this reason, the function with new features was proposed with new features to filter the data correctly without any errors.

This function is simple, but it is important to standardize the beats number for any researcher that are used PhysioNet databases. This function can be added to the MATLAB-WFDB toolbox to simply filtered the annotations files by removing the non-beat annotations precisely with the standard values. The function read and search all annotations data files for each record, as shown in Figure 1. If the annotation is one of the non-beat types, this annotation will be removed from the annotation data. Also, it has to be used for any PhysioNet database to extract the beat annotation by removing the non-beat annotations used to prepare the data for many applications, including QRS-detection methods.

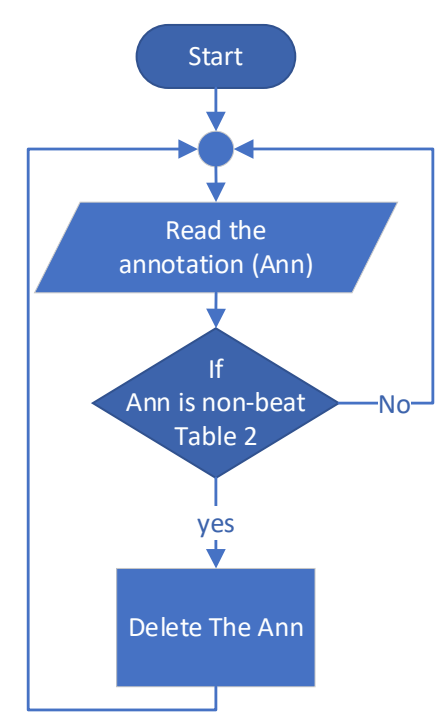

Figure 1. The function flowchart

\section{THE COMPARISON AND VERIFICATION RESULTS WITH DISCUSSION}

The work focuses on the verification and comparison of the MIT-BIH arrhythmia database used for the QRS-detection algorithm. The proposed heartbeats filter function can apply to all MIT-BIH databases from the PhysioNet site. The reviewed QRS-detection methods are not using the same number of heartbeats for the MIT-BIH arrhythmia database. This number should be standard for this database because it depends on the original database's beats number. Simultaneously, not all the QRS-detection methods are considering the same number of beats for the same database records. The revision for the existing QRS-detection methods using the MIT-BIH arrhythmia database has summarized the errors for these methods based on the beats for records shown in Table 4 (see in Appendix). The incorrect records are indicated by bold, the Total $(\mathrm{T})$, and Errors (E) in this table. The methods should use the same number of beats without any difference, but the errors are occurring by researchers. All the reviewed methods are revised, compared, and verified based on beats number for each database record. Table 5 summarizes the total beats number, total error per record, and total error per database for different methods to evaluate these methods' incorrectness.

The total number of beats for the MIT-BIH arrhythmia database used from the reviewed methods is calculated; this number should be 109,494 heartbeats for all database records, as shown in section 2 . The beat errors for these methods compared to the correct number of beats for this database are determined to find the number of methods that used the correct beat's value. Also, the other methods contained errors start from 1 beat to 1400 beats for the overall database. Table 5 shows the percentage of references number for each error per the total references that were reviewed. Moreover, it shows the total number of errors for each reference per each record (sum of the absolute values of errors) and the total number of errors for each reference per overall database, which takes a positive or negative value.

The beats errors per all data record up to 1400 beats and $29 \%$ of the total reviewed methods use the correct beats number. On the other hand, $71 \%$ are using incorrect beats number. Also, the number of incorrect methods is higher than the number of correct methods based on our comparison. So, we propose this study. Each record in the database for the reviewed methods has been studied for beats errors calculation. 
Figure 2 describes the number of references that contain errors for each record. For each record, the percentage of the reference number that occurs error per all references is calculated to evaluate the record error reasons. The records error percentage is started from 53\% for record no. 207 to $9 \%$ for records (102, $103,112,117,119,122,123$, and 230).

Table 5 and Figure 2 show the difference between these methods for the same records used from the same database. After the results are studied, the following obvious points are established:

a. The correct number of the beats is 109,494 beats without adding or removing any data.

b. The designed function extracts the correct heartbeats number of all records for the MIT-BIH arrhythmia database.

c. If the beats number exceed the correct number:

- Some non-beat annotations have been added and should be mentioned in the methods.

- The data has been repeated for record and should be mentioned in the methods.

d. If the beats number less than the correct number:

- Some beat annotations have been removed and should be mentioned in the methods.

e. This database contains some errors before digitalization and verification [1].

f. The WFDB toolbox does not include the beats or non-beats filter for the (rdann) function that reads the annotations files.

g. The copy and paste records beat numbers between the researchers without verification.

h. A high number of annotation types (39 annotations) confuse the researchers.

i. According to Figure 2, the most error occurs in record no. 207 because many researchers are counting the 472 ventricular flutter waves, but these waves are considered as non-beat annotations based on the annotation's description of PhysioNet.

j. From Figure 2, records no. 209 is the second, and records no. 214 is the third most errors beats for the reviewed methods, but the number of errors is low and not exceeds eight beats and nine beats, respectively.

k. According to Figure 2, the lowest error records $(102,103,112,117,119,122,123$, and 230) because these records contain the lowest non-beat annotations.

Table 5. Total beat annotations and errors for the reviewed methods

\begin{tabular}{|c|c|c|c|c|c|}
\hline References & Count of ref & $\begin{array}{l}\text { Percentage } \\
\text { ref }\end{array}$ & Total beats & Total errors per record & $\begin{array}{c}\text { Total Errors per } \\
\text { database }\end{array}$ \\
\hline$[5-24]$ & 20 & $29 \%$ & 109494 & 0 & 0 \\
\hline$[25]$ & 1 & $1 \%$ & 109493 & 1 & -1 \\
\hline [26-31] & 6 & $9 \%$ & 109496 & 2 & 2 \\
\hline$[32]$ & 1 & $1 \%$ & 109495 & 3 & 1 \\
\hline [33] & 1 & $1 \%$ & 109488 & 6 & -6 \\
\hline [34] & 1 & $1 \%$ & 109488 & 6 & -6 \\
\hline$[35]$ & 1 & $1 \%$ & 109486 & 8 & -8 \\
\hline$[36]$ & 1 & $1 \%$ & 109481 & 13 & -13 \\
\hline [37-39] & 3 & $4 \%$ & 109508 & 16 & 14 \\
\hline$[40-42]$ & 3 & $4 \%$ & 109510 & 18 & 16 \\
\hline$[43]$ & 1 & $1 \%$ & 109483 & 23 & -11 \\
\hline [44] & 1 & $1 \%$ & 109488 & 36 & -6 \\
\hline$[45]$ & 1 & $1 \%$ & 109478 & 44 & -16 \\
\hline [46] & 1 & $1 \%$ & 109443 & 51 & -51 \\
\hline [47] & 1 & $1 \%$ & 109428 & 66 & -66 \\
\hline [48] & 1 & $1 \%$ & 109328 & 166 & -166 \\
\hline [49] & 1 & $1 \%$ & 109357 & 203 & -137 \\
\hline$[50]$ & 1 & $1 \%$ & 109255 & 239 & -239 \\
\hline [51-56] & 6 & $9 \%$ & 109809 & 329 & 315 \\
\hline [57] & 1 & $1 \%$ & 109788 & 348 & 294 \\
\hline [58] & 1 & $1 \%$ & 109267 & 357 & -227 \\
\hline [59] & 1 & $1 \%$ & 109134 & 360 & -360 \\
\hline [60] & 1 & $1 \%$ & 109097 & 423 & -397 \\
\hline [61-63] & 3 & $4 \%$ & 109966 & 472 & 472 \\
\hline [64] & 1 & $1 \%$ & 109965 & 473 & 471 \\
\hline [65] & 1 & $1 \%$ & 109966 & 474 & 472 \\
\hline [66] & 1 & $1 \%$ & 109996 & 502 & 502 \\
\hline [67] & 1 & $1 \%$ & 109369 & 567 & -125 \\
\hline [68] & 1 & $1 \%$ & 109985 & 579 & 491 \\
\hline [69] & 1 & $1 \%$ & 109663 & 603 & 169 \\
\hline$[70]$ & 1 & $1 \%$ & 110159 & 665 & 665 \\
\hline [71] & 1 & $1 \%$ & 110008 & 738 & 514 \\
\hline [72] & 1 & $1 \%$ & 109036 & 1400 & -458 \\
\hline Total & 68 & $100 \%$ & & & \\
\hline
\end{tabular}




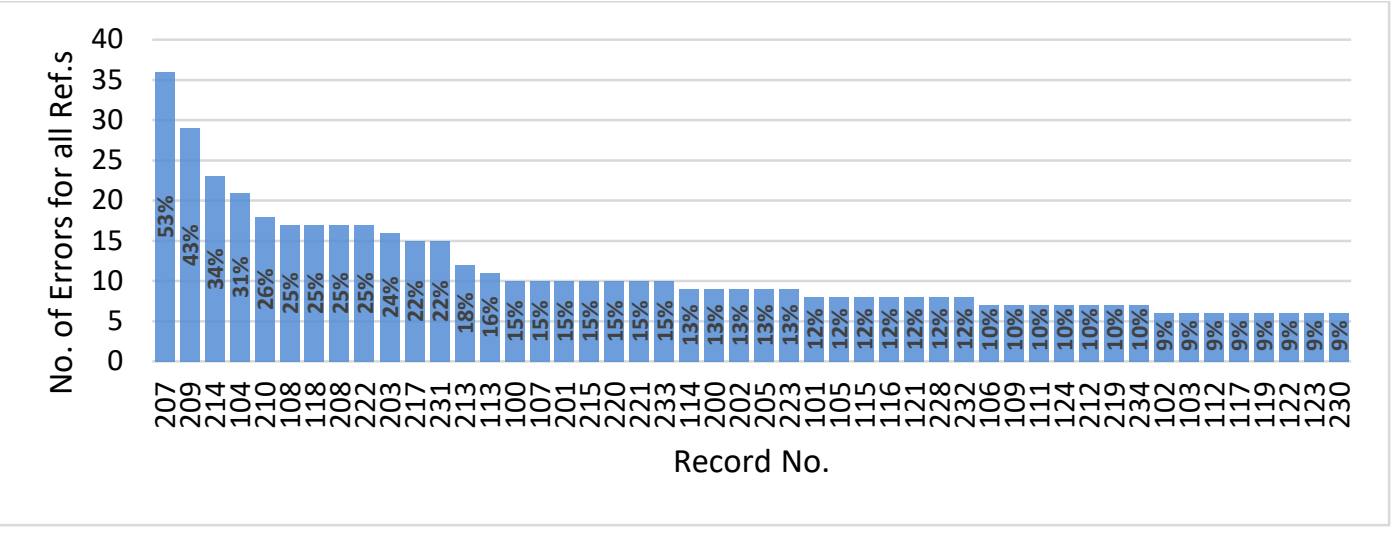

Figure 2. Records errors per overall references

\section{CONCLUSION}

This paper presented a method for finding the correct beats number for the MIT-BIH arrhythmia database with a comparison study and design a function for MATLAB to extract the correct values for any PhysioNet databases. In this way, the number of beats that are using by the researchers will be standards. The non-beat annotations affected the results of the QRS-detection methods in two ways. First, the proposed methods' evaluation accuracy is not calculated correctly because the number of database beats is incorrect. Second, the methods based on machine learning are trained depending on incorrect information. So, the learning operation was not proper, and the results of the methods are not correct. Most reviewed methods used an incorrect number of beats, $29 \%$ of researchers used the correct number, and $71 \%$ are used incorrect beats. The proposed function should be added to the MATLAB-WFDB Toolbox to filter the annotations files to remove the non-beat annotations correctly and extract the standard beat values. It can be used for any other programing language to read the annotations files from the PhysioNet databases like python.

\section{APPENDIX}

Table 4. The beat annotation for the reviewed methods

\begin{tabular}{|c|c|c|c|c|c|c|c|c|c|c|c|c|c|c|c|c|c|}
\hline Rec & {$[5-24]$} & {$[25]$} & [26-30] & [31] & [32] & [33] & {$[34]$} & {$[35]$} & [36] & [37-39] & [40-42] & [43] & [44] & {$[45]$} & {$[46]$} & [47] & [48] \\
\hline 100 & 2273 & 2273 & 2273 & 2273 & 2273 & 2273 & 2272 & 2272 & 2272 & 2273 & 2273 & 2273 & 2273 & 2273 & 2271 & 2273 & 2272 \\
\hline 101 & 1865 & 1865 & 1865 & 1865 & 1865 & 1865 & 1865 & 1865 & 1865 & 1865 & 1865 & 1865 & 1865 & 1865 & 1864 & 1865 & 1864 \\
\hline 102 & 2187 & 2187 & 2187 & 2187 & 2187 & 2187 & 2187 & 2187 & 2187 & 2187 & 2187 & 2187 & 2187 & 2187 & 2186 & 2187 & 2187 \\
\hline 103 & 2084 & 2084 & 2084 & 2084 & 2084 & 2084 & 2084 & 2084 & 2084 & 2084 & 2084 & 2084 & 2084 & 2084 & 2083 & 2084 & 2084 \\
\hline 104 & 2229 & 2229 & 2229 & 2229 & 2229 & 2229 & 2228 & 2228 & 2228 & 2229 & 2229 & 2229 & 2229 & 2230 & 2228 & 2229 & 2227 \\
\hline 105 & 2572 & 2572 & 2572 & 2572 & 2572 & 2572 & 2572 & 2572 & 2572 & 2572 & 2572 & 2572 & 2572 & 2572 & 2571 & 2572 & 2555 \\
\hline 106 & 2027 & 2027 & 2027 & 2027 & 2027 & 2027 & 2027 & 2027 & 2027 & 2027 & 2027 & 2027 & 2027 & 2027 & 2026 & 2027 & 2027 \\
\hline 107 & 2137 & 2136 & 2137 & 2137 & 2137 & 2137 & 2136 & 2137 & 2137 & 2137 & 2137 & 2137 & 2137 & 2137 & 2136 & 2137 & 2135 \\
\hline 108 & 1763 & 1763 & 1763 & 1763 & 1763 & 1763 & 1763 & 1763 & 1763 & 1774 & 1774 & 1760 & 1774 & 1763 & 1762 & 1763 & 1761 \\
\hline 109 & 2532 & 2532 & 2532 & 2532 & 2532 & 2532 & 2532 & 2532 & 2532 & 2532 & 2532 & 2532 & 2532 & 2532 & 2531 & 2532 & 2532 \\
\hline 111 & 2124 & 2124 & 2124 & 2124 & 2124 & 2124 & 2124 & 2124 & 2124 & 2124 & 2124 & 2124 & 2124 & 2124 & 2123 & 2124 & 2124 \\
\hline 112 & 2539 & 2539 & 2539 & 2539 & 2539 & 2539 & 2539 & 2539 & 2539 & 2539 & 2539 & 2539 & 2539 & 2539 & 2538 & 2539 & 2539 \\
\hline 113 & 1795 & 1795 & 1795 & 1795 & 1795 & 1795 & 1794 & 1794 & 1794 & 1795 & 1795 & 1795 & 1795 & 1795 & 1793 & 1795 & 1794 \\
\hline 114 & 1879 & 1879 & 1879 & 1879 & 1879 & 1879 & 1879 & 1878 & 1879 & 1879 & 1879 & 1872 & 1879 & 1879 & 1878 & 1879 & 1879 \\
\hline 115 & 1953 & 1953 & 1953 & 1953 & 1953 & 1953 & 1953 & 1953 & 1953 & 1953 & 1953 & 1952 & 1953 & 1953 & 1952 & 1953 & 1952 \\
\hline 116 & 2412 & 2412 & 2412 & 2412 & 2412 & 2412 & 2412 & 2412 & 2412 & 2412 & 2412 & 2412 & 2412 & 2412 & 2411 & 2412 & 2410 \\
\hline 117 & 1535 & 1535 & 1535 & 1535 & 1535 & 1535 & 1535 & 1535 & 1535 & 1535 & 1535 & 1535 & 1535 & 1535 & 1534 & 1535 & 1535 \\
\hline 118 & 2278 & 2278 & 2278 & 2278 & 2278 & 2278 & 2278 & 2278 & 2277 & 2278 & 2278 & 2278 & 2278 & 2288 & 2277 & 2278 & 2278 \\
\hline 119 & 1987 & 1987 & 1987 & 1987 & 1987 & 1987 & 1987 & 1987 & 1987 & 1987 & 1987 & 1987 & 1987 & 1987 & 1986 & 1987 & 1987 \\
\hline 121 & 1863 & 1863 & 1863 & 1863 & 1863 & 1863 & 1863 & 1862 & 1862 & 1863 & 1863 & 1863 & 1863 & 1863 & 1862 & 1863 & 1863 \\
\hline 122 & 2476 & 2476 & 2476 & 2476 & 2476 & 2476 & 2476 & 2476 & 2476 & 2476 & 2476 & 2476 & 2476 & 2476 & 2475 & 2476 & 2476 \\
\hline 123 & 1518 & 1518 & 1518 & 1518 & 1518 & 1518 & 1518 & 1518 & 1518 & 1518 & 1518 & 1518 & 1518 & 1518 & 1517 & 1518 & 1518 \\
\hline 124 & 1619 & 1619 & 1619 & 1619 & 1619 & 1619 & 1619 & 1619 & 1619 & 1619 & 1619 & 1619 & 1619 & 1619 & 1618 & 1619 & 1619 \\
\hline 200 & 2601 & 2601 & 2601 & 2601 & 2601 & 2601 & 2601 & 2601 & 2601 & 2601 & 2601 & 2601 & 2601 & 2601 & 2600 & 2601 & 2581 \\
\hline 201 & 1963 & 1963 & 1963 & 1963 & 1963 & 1963 & 1963 & 1963 & 1962 & 1963 & 1963 & 1963 & 1963 & 1963 & 1962 & 1963 & 1950 \\
\hline 202 & 2136 & 2136 & 2136 & 2136 & 2136 & 2135 & 2136 & 2136 & 2136 & 2136 & 2136 & 2136 & 2136 & 2136 & 2135 & 2136 & 2133 \\
\hline 203 & 2980 & 2980 & 2980 & 2980 & 2980 & 2980 & 2980 & 2980 & 2980 & 2980 & 2980 & 2980 & 2980 & 2980 & 2979 & 2980 & 2949 \\
\hline 205 & 2656 & 2656 & 2656 & 2656 & 2656 & 2656 & 2656 & 2656 & 2655 & 2656 & 2656 & 2656 & 2656 & 2656 & 2655 & 2656 & 2647 \\
\hline 207 & 1860 & 1860 & 1862 & 1860 & 1862 & 1860 & 1860 & 1860 & 1860 & 1860 & 1862 & 1862 & 1860 & 1862 & 1859 & 1794 & 1859 \\
\hline
\end{tabular}


Table 4. The beat annotation for the reviewed methods (continue)

\begin{tabular}{|c|c|c|c|c|c|c|c|c|c|c|c|c|c|c|c|c|c|}
\hline Rec & {$[5-24]$} & [25] & {$[26-30]$} & [31] & [32] & [33] & [34] & [35] & [36] & [37-39] & [40-42] & [43] & [44] & [45] & [46] & [47] & [48] \\
\hline 210 & 2650 & 2650 & 2650 & 2650 & 2650 & 2650 & 2650 & 2650 & 2650 & 2650 & 2650 & 2650 & 2650 & 2647 & 2649 & 2650 & 2644 \\
\hline 213 & 3251 & 3251 & 3251 & 3251 & 3251 & 3251 & 3250 & 3250 & 3250 & 3251 & 3251 & 3251 & 3251 & 3251 & 3249 & 3251 & 3249 \\
\hline 214 & 2262 & 2262 & 2262 & 2261 & 2261 & 2261 & 2262 & 2261 & 2261 & 2265 & 2265 & 2265 & 2265 & 2254 & 2261 & 2262 & 2261 \\
\hline 215 & 3363 & 3363 & 3363 & 3363 & 3363 & 3361 & 3363 & 3363 & 3363 & 3363 & 3363 & 3363 & 3363 & 3353 & 3362 & 3363 & 3362 \\
\hline 217 & 2208 & 2208 & 2208 & 2208 & 2208 & 2208 & 2208 & 2208 & 2208 & 2209 & 2209 & 2209 & 2209 & 2208 & 2207 & 2208 & 2208 \\
\hline 221 & 2427 & 2427 & 2427 & 2427 & 2427 & 2427 & 2427 & 2427 & 2426 & 2427 & 2427 & 2427 & 2407 & 2427 & 2426 & 2427 & 2427 \\
\hline 222 & 2483 & 2483 & 2483 & 2483 & 2483 & 2483 & 2483 & 2483 & 2483 & 2483 & 2483 & 2483 & 2483 & 2484 & 2482 & 2483 & 2482 \\
\hline 223 & 2605 & 2605 & 2605 & 2605 & 2605 & 2605 & 2605 & 2605 & 2605 & 2605 & 2605 & 2605 & 2605 & 2605 & 2604 & 2605 & 2603 \\
\hline 228 & 2053 & 2053 & 2053 & 2053 & 2053 & 2053 & 2053 & 2053 & 2053 & 2053 & 2053 & 2048 & 2053 & 2053 & 2052 & 2053 & 2053 \\
\hline 230 & 2256 & 2256 & 2256 & 2256 & 2256 & 2256 & 2256 & 2256 & 2256 & 2256 & 2256 & 2256 & 2256 & 2256 & 2255 & 2256 & 2256 \\
\hline 231 & 1571 & 1571 & 1571 & 1571 & 1571 & 1571 & 1571 & 1571 & 1571 & 1571 & 1571 & 1571 & 1571 & 1571 & 1570 & 1571 & 1571 \\
\hline
\end{tabular}

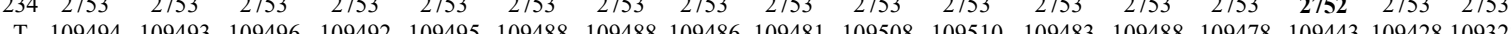

\begin{tabular}{|c|c|c|c|c|c|c|c|c|c|c|c|c|c|c|c|c|c|}
\hline & & & & & & & & & & & & & & & & & \\
\hline E & 0 & 1 & 2 & 2 & 3 & 6 & 6 & 8 & 13 & 16 & 18 & 23 & 36 & 44 & 51 & 66 & 166 \\
\hline Rec & [49] & [50] & {$[51-56]$} & [57] & [58] & [59] & {$[60]$} & [61-63] & [64] & [65] & [66] & [67] & [68] & [69] & [70] & [71] & [72] \\
\hline 100 & 2269 & 2273 & 2273 & 2273 & 2267 & 2265 & 2273 & 2273 & 2273 & 2273 & 2273 & 2273 & 2274 & 2270 & 2273 & 2273 & 2272 \\
\hline 101 & 1862 & 1864 & 1865 & 1865 & 1859 & 1860 & 1865 & 1865 & 1865 & 1865 & 1865 & 1865 & 1866 & 1862 & 1865 & 1873 & 1864 \\
\hline 102 & 2183 & 2187 & 2187 & 2187 & 2181 & 2180 & 2187 & 2187 & 2187 & 2187 & 2187 & 2187 & 2187 & 2186 & 2187 & 2186 & 2186 \\
\hline 103 & 2081 & 2084 & 2084 & 2084 & 2081 & 2078 & 2084 & 2084 & 2084 & 2084 & 2084 & 2084 & 2084 & 2083 & 2084 & 2084 & 2083 \\
\hline 104 & 2225 & 2226 & 2230 & 2230 & 2224 & 2222 & 2230 & 2229 & 2229 & 2230 & 2229 & 2229 & 2229 & 2219 & 2229 & 2235 & 2228 \\
\hline 105 & 2582 & 2566 & 2572 & 2572 & 2564 & 2565 & 2572 & 2572 & 2572 & 2572 & 2572 & 2572 & 2602 & 2559 & 2572 & 2578 & 2571 \\
\hline 106 & 2024 & 2023 & 2027 & 2027 & 2024 & 2021 & 2027 & 2027 & 2027 & 2027 & 2027 & 2027 & 2026 & 2025 & 2027 & 2096 & 2026 \\
\hline 107 & 2133 & 2135 & 2137 & 2137 & 2131 & 2131 & 2137 & 2137 & 2137 & 2137 & 2137 & 2137 & 2136 & 2135 & 2137 & 2138 & 2136 \\
\hline 108 & 1761 & 1759 & 1763 & 1763 & 1757 & 1757 & 1763 & 1763 & 1763 & 1763 & 1763 & 1763 & 1763 & 1747 & 1774 & 1763 & 1762 \\
\hline 109 & 2528 & 2527 & 2532 & 2532 & 2526 & 2524 & 2532 & 2532 & 2532 & 2532 & 2532 & 2532 & 2533 & 2531 & 2532 & 2519 & 1649 \\
\hline 111 & 2121 & 2123 & 2124 & 2124 & 2120 & 2118 & 2124 & 2124 & 2124 & 2124 & 2124 & 2124 & 2123 & 2120 & 2124 & 2124 & 2123 \\
\hline 112 & 2535 & 2539 & 2539 & 2539 & 2536 & 2531 & 2539 & 2539 & 2539 & 2539 & 2539 & 2539 & 2539 & 2537 & 2539 & 2549 & 2538 \\
\hline 113 & 1791 & 1795 & 1795 & 1797 & 1791 & 1789 & 1795 & 1795 & 1795 & 1795 & 1795 & 1795 & 1794 & 1792 & 1795 & 1795 & 1794 \\
\hline 114 & 1875 & 1832 & 1879 & 1879 & 1872 & 1872 & 1879 & 1879 & 1879 & 1879 & 1879 & 79 & 1890 & & 1879 & 1885 & 1878 \\
\hline 115 & 1949 & 1953 & 1953 & 1953 & 1945 & 1946 & 1953 & & & 1953 & & & 1953 & & 1953 & 1960 & 1952 \\
\hline 116 & 2408 & 2392 & 2412 & 2412 & 2409 & 2404 & 2412 & 2412 & 2412 & 2412 & 2 & 2 & 2395 & & & 2401 & 2411 \\
\hline 117 & 1532 & 1535 & 1535 & 1535 & 1532 & 1530 & 1535 & 1535 & 1535 & 1535 & 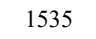 & 1 & 1535 & & 1535 & 1538 & 1534 \\
\hline 118 & 2275 & 2278 & 2275 & 2275 & 2273 & 2271 & 2275 & 2278 & 2278 & 2278 & 0 & 2 & 2278 & 2275 & 2288 & 2298 & 2277 \\
\hline 119 & 1984 & 1987 & 1987 & 1987 & 1985 & 1981 & 1987 & 1987 & 1987 & 1987 & 87 & 1 & 1988 & 1985 & 1987 & 2010 & 1986 \\
\hline 121 & 1859 & 1863 & 1863 & 1863 & 1858 & 1856 & 1863 & 1863 & 1863 & 1863 & 63 & 1 & 1863 & 18 & 1863 & 1871 & 1862 \\
\hline 122 & 2472 & 2476 & 2476 & 2476 & 2471 & 2468 & 2476 & 2476 & 2476 & 2476 & 2476 & 2476 & 2476 & 2475 & 2476 & 2477 & 2475 \\
\hline 123 & 1515 & 1518 & 1518 & 1518 & 1514 & 1513 & 1518 & 1518 & 1518 & 1518 & 1518 & 1518 & 1519 & 1517 & 1518 & 1518 & 1517 \\
\hline 124 & 1616 & 1619 & 1619 & 1619 & 1613 & 1613 & 1619 & 16 & 1619 & 1618 & 9 & 1 & 1619 & 16 & 1619 & 1602 & 1618 \\
\hline 200 & 2597 & 2600 & 2601 & 2601 & 2595 & 2593 & 2607 & 2601 & 2601 & 2601 & 2601 & & 2601 & 2560 & 2601 & 2599 & 2600 \\
\hline 201 & 1961 & 1934 & 1963 & 1963 & 1946 & 1959 & 1963 & & 1963 & 19 & & & & & 2000 & 1963 & 1962 \\
\hline 202 & 2132 & 2132 & 2136 & 213 & 2134 & 2128 & 2136 & 213 & 2136 & & & & & & & 2135 & 2135 \\
\hline 203 & 3003 & 2926 & 2982 & 297 & 2976 & 2973 & 2982 & 2980 & 2980 & 298 & 2980 & & 2988 & & 2980 & 2982 & 2979 \\
\hline 205 & 2652 & 2653 & 2656 & 2656 & 2650 & 2648 & 2656 & 2656 & 2656 & 2656 & 6 & 2 & 2656 & 2654 & 2656 & 2657 & 2655 \\
\hline 207 & 1855 & 1857 & 1862 & 1862 & 1856 & 1850 & 1862 & 2332 & 2332 & 2332 & 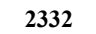 & 1543 & 2324 & 2246 & 2332 & 1862 & 2331 \\
\hline 208 & 2951 & 2940 & 2956 & 2954 & 2953 & 2946 & 2956 & 2955 & 2955 & 2955 & 55 & 29 & 2953 & 2937 & 2955 & 2952 & 2954 \\
\hline 209 & 3001 & 3005 & 3004 & 3004 & 2999 & 2997 & 3004 & 3005 & 3004 & 3005 & 3005 & 3006 & 3006 & 3002 & 3005 & 3051 & 3004 \\
\hline 210 & 2646 & 2628 & 2647 & 2647 & 2645 & 2642 & 2647 & 2650 & 2650 & 2650 & 2650 & 2640 & 2652 & 2640 & 2650 & 2645 & 2649 \\
\hline 212 & 2744 & 2748 & 2748 & 2748 & 2746 & 2740 & 2748 & 2748 & 2748 & 2748 & 2748 & 2748 & 2748 & 2746 & 2748 & 2761 & 2747 \\
\hline 213 & 3246 & 3250 & 3251 & 3251 & 3245 & 3241 & 3251 & 3251 & 3251 & 3251 & 3251 & 34 & 3250 & 32 & 3251 & 3245 & 3250 \\
\hline 214 & 2258 & 2258 & 2 & 226 & 2255 & 22 & 22 & & 22 & 22 & & & 22 & & 2 & 2273 & 2261 \\
\hline 215 & 3358 & 336 & & 33 & 33 & 33 & & & & & & & & & & 3398 & 3362 \\
\hline 217 & 2205 & 2207 & 22 & 2208 & 2202 & 2202 & 2208 & 22 & 2208 & 2208 & $2 \angle 08$ & 2208 & 2208 & & 2208 & 2270 & 2207 \\
\hline 219 & 2151 & 2154 & 2154 & 2154 & 2150 & 2147 & 2154 & 2154 & 2154 & 2154 & 2154 & 2154 & 2154 & 2152 & 2287 & 2154 & 2153 \\
\hline 220 & 2044 & 2047 & 2048 & 2048 & 2041 & 2041 & 2048 & 2048 & 2048 & 2048 & 2048 & 2048 & 2048 & 2047 & 2048 & 2068 & 2047 \\
\hline 221 & 2423 & 2426 & 2427 & 2427 & 2422 & 2420 & 2427 & 2427 & 2427 & 2427 & 2457 & 2427 & 2427 & 2426 & 2427 & 2447 & 2426 \\
\hline 222 & 2478 & 2481 & 2484 & 2484 & 2492 & 2474 & 2484 & 2483 & 2483 & 2483 & 2483 & 2483 & 2485 & 2481 & 2483 & 2624 & 2482 \\
\hline 223 & 2601 & 2604 & 2605 & 2605 & 2603 & 2581 & 2605 & 2605 & 2605 & 2605 & 2605 & 2589 & 2604 & 2604 & 2605 & 2636 & 2604 \\
\hline 228 & 2050 & 2050 & 2053 & 2053 & 2048 & 2047 & 2053 & 2053 & 2053 & 2053 & 2053 & 2053 & 2060 & 2051 & 2053 & 2116 & 2052 \\
\hline 230 & 2252 & 2256 & 2256 & 2256 & 2252 & 2248 & 2256 & 2256 & 2256 & 2256 & 2256 & 2256 & 2256 & 22 & 2256 & 2257 & 2255 \\
\hline 231 & 1568 & 1571 & & 188 & 1566 & 1565 & 1186 & & & 1571 & & & & & & 1569 & 1570 \\
\hline 232 & 1778 & 1780 & 1780 & 1767 & 1719 & 1776 & 1780 & & 1780 & 1780 & 1780 & 1780 & 1783 & 1779 & 1780 & 1734 & 1779 \\
\hline 233 & 3074 & 3078 & 3079 & 3076 & 3135 & 3069 & 3079 & 3079 & 3079 & 3079 & 3079 & 3079 & 3077 & 3076 & 3079 & 3074 & 3078 \\
\hline 234 & 2749 & 2753 & 2753 & 2753 & 2747 & 2745 & 2735 & 2753 & 2753 & 2753 & 2753 & 2753 & 2751 & 2751 & 2753 & 2763 & 2752 \\
\hline $\mathrm{T}$ & 109357 & 109255 & 109809 & 109788 & 109267 & 109134 & 109097 & 109966 & 109965 & 109966 & 109996 & 109369 & 109985 & 109663 & 110159 & 110008 & 109036 \\
\hline E & 203 & 239 & 329 & 348 & 357 & 360 & 423 & 472 & 473 & 474 & 502 & 567 & 579 & 603 & 665 & 738 & 1400 \\
\hline
\end{tabular}




\section{REFERENCES}

[1] S. Mendis et al., "Global atlas on cardiovascular disease prevention and control," Geneva: World Health Organization in Collaboration with The World Heart Federation and the World Stroke Organization, 2011.

[2] S. K. Berkaya, A. K. Uysal, E. S. Gunal, S. Ergin, S. Gunal, and M. B. Gulmezoglu, "A survey on ECG analysis," Biomedical Signal Processing and Control, vol. 43, pp. 216-235, May 2018, doi: 10.1016/j.bspc.2018.03.003.

[3] G. B. Moody and R. G. Mark, "The impact of the MIT-BIH Arrhythmia Database," in IEEE Engineering in Medicine and Biology Magazine, vol. 20, no. 3, pp. 45-50, May-June 2001, doi: 10.1109/51.932724.

[4] I. Silva and G. B. Moody, "An Open-source Toolbox for Analysing and Processing PhysioNet Databases in MATLAB and Octave," Journal of Open Research Software, vol. 2, no.1, pp. 1-27, Sep. 2014, doi: 10.5334/jors.bi.

[5] A. J. Khalaf and S. J. Mohammed, "A QRS-Detection Algorithm for Real-Time Applications," International Journal of Intelligent Engineering and Systems, vol. 14, no. 1, pp. 356-367, 2020, doi: 10.22266/ijies2021.0228.33.

[6] C. Nayak, S. K. Saha, R. Kar and D. Mandal, "An Efficient and Robust Digital Fractional Order Differentiator Based ECG Pre-Processor Design for QRS Detection," in IEEE Transactions on Biomedical Circuits and Systems, vol. 13, no. 4, pp. 682-696, Aug. 2019, doi: 10.1109/TBCAS.2019.2916676.

[7] A. Sharma, S. Patidar, A. Upadhyay, and U. R. Acharya, "Accurate tunable-Q wavelet transform based method for QRS complex detection," Computers \& Electrical Engineering, vol. 75, pp. 101-111, May 2019, doi: 10.1016/j.compeleceng.2019.01.025.

[8] A. Bajaj and S. Kumar, "QRS complex detection using fractional Stockwell transform and fractional Stockwell Shannon energy," Biomedical Signal Processing and Control, vol. 54, Sep. 2019, Art. no. 101628, doi: 10.1016/j.bspc.2019.101628.

[9] T. Nguyen, X. Qin, A. Dinh, and F. Bui, "Low Resource Complexity R-peak Detection Based on Triangle Template Matching and Moving Average Filter," Sensors, vol. 19, no. 18, Sep. 2019, Art. no. 3997, doi: $10.3390 / \mathrm{s} 19183997$.

[10] C. Nayak, S. K. Saha, R. Kar, and D. Mandal, "Optimal SSA-based wideband digital differentiator design for cardiac QRS complex detection application," International Journal of Numerical Modelling: Electronic Networks, Devices and Fields, vol. 32, no. 2, Nov. 2018, Art. no. e2524, doi: 10.1002/jnm.2524.

[11] Ö. Yakut and E. D. Bolat, "An improved QRS complex detection method having low computational load," Biomedical Signal Processing and Control, vol. 42, pp. 230-241, Apr. 2018, doi: 10.1016/j.bspc.2018.02.004.

[12] D. Yang and Y. Zhang, "A Real-time QRS Detector Based on Low-pass Differentiator and Hilbert Transform," in MATEC Web of Conferences, Guangzhou, China, vol. 175, pp. 1-6, 2018, doi: 10.1051/matecconf/201817502008.

[13] T. Sharma and K. K. Sharma, "QRS complex detection in ECG signals using locally adaptive weighted total variation denoising," Computers in Biology and Medicine, vol. 87, pp. 187-199, Aug. 2017, doi: 10.1016/j.compbiomed.2017.05.027.

[14] S. Jain, A. Kumar, and V. Bajaj, "Technique for QRS complex detection using particle swarm optimisation," IET Science, Measurement \& Technology, vol. 10, no. 6, pp. 626-636, Sep. 2016, doi: 10.1049/iet-smt.2016.0023.

[15] T. Sharma and K. K. Sharma, "QRS Complex Detection in ECG Signals Using the Synchrosqueezed Wavelet Transform," IETE Journal of Research, vol. 62, no. 6, pp. 885-892, Sep. 2016, doi: 10.1080/03772063.2016.1221744.

[16] S. Yazdani and J. Vesin, "Extraction of QRS fiducial points from the ECG using adaptive mathematical morphology," Digital Signal Processing, vol. 56, pp. 100-109, Sep. 2016, doi: 10.1016/j.dsp.2016.06.010.

[17] D. Castells-Rufas and J. Carrabina, "Simple real-time QRS detector with the MaMeMi filter," Biomedical Signal Processing and Control, vol. 21, pp. 137-145, Aug. 2015, doi: 10.1016/j.bspc.2015.06.001.

[18] F. Bouaziz, D. Boutana, and M. Benidir, "Multiresolution wavelet-based QRS complex detection algorithm suited to several abnormal morphologies," IET Signal Processing, vol. 8, no. 7, pp. 774-782, Sep. 2014, doi: 10.1049/ietspr.2013.0391.

[19] G. Nallathambi and J. C. Príncipe, "Integrate and Fire Pulse Train Automaton for QRS detection," in IEEE Transactions on Biomedical Engineering, vol. 61, no. 2, pp. 317-326, Feb. 2014, doi: 10.1109/TBME.2013.2282954.

[20] Y. Li, F. Hong, and J. Song, "A new approach of QRS complex detection based on matched filtering and triangle character analysis," Australasian Physical \& Engineering Sciences in Medicine, vol. 35, no. 3, pp. 341-356, Jul. 2012, doi: 10.1007/s13246-012-0149-x.

[21] Z. Zidelmal, A. Amirou, M. Adnane, and A. Belouchrani., "QRS detection based on wavelet coefficients," Computer Methods and Programs in Biomedicine, vol. 107, no. 3, pp. 490-496, Sep. 2012, doi: 10.1016/j.cmpb.2011.12.004.

[22] M. Adnane, Z. Jiang, and S. Choi., "Development of QRS detection algorithm designed for wearable cardiorespiratory system," Computer Methods and Programs in Biomedicine, vol. 93, no. 1, pp. 20-31, Jan. 2009, doi: 10.1016/j.cmpb.2008.07.010.

[23] F. Chiarugi, V. Sakkalis, D. Emmanouilidou, T. Krontiris, M. Varanini and I. Tollis, "Adaptive threshold QRS detector with best channel selection based on a noise rating system," 2007 Computers in Cardiology, 2007, pp. 157-160, doi: 10.1109/CIC.2007.4745445.

[24] M. Cvikl, F. Jager, and Z. Andrej, "Hardware Implementation of a Modified Delay-Coordinate Mapping-Based QRS Complex Detection Algorithm," EURASIP Journal on Advances in Signal Processing, vol. 2007, no. 1, Apr. 2007, doi: 10.1155/2007/57286. 
[25] M. Elgendi, M. Jonkman, and F. De Boer, "Frequency Bands Effects On QRS Detection," in Proceedings of the Third International Conference on Bio-inspired Systems and Signal Processing, Valencia, Spain, vol. 1, pp. 428-431, 2010, doi: 10.5220/0002742704280431.

[26] M. S. Manikandan and B. Ramkumar, "Straightforward and robust QRS detection algorithm for wearable cardiac monitor," Healthcare Technology Letters, vol. 1, no. 1, pp. 40-44, Jan. 2014, doi: 10.1049/htl.2013.0019.

[27] M. S. Manikandan and K. P. Soman, "A novel method for detecting R-peaks in electrocardiogram (ECG) signal," Biomedical Signal Processing and Control, vol. 7, no. 2, pp. 118-128, Mar. 2012, doi: 10.1016/j.bspc.2011.03.004.

[28] J. Lewandowski, H. E. Arochena, R. N. G. Naguib and K. Chao, "A simple real-time QRS detection algorithm utilizing curve-length concept with combined adaptive threshold for electrocardiogram signal classification," TENCON 2012 IEEE Region 10 Conference, 2012, pp. 1-6, doi: 10.1109/TENCON.2012.6412176.

[29] N. S. V. K. Chaitanya, A. Radhakrishnan, G. R. Reddy and M. S. Manikandan, "A simple and robust QRS detection algorithm for wireless medical body area network," 2011 International Conference on Emerging Trends in Networks and Computer Communications (ETNCC), 2011, pp. 153-158, doi: 10.1109/ETNCC.2011.5958505.

[30] B. Huang and Y. Wang, "Detecting QRS Complexes of Two-Channel ECG Signals by Using Combined Wavelet Entropy," 2009 3rd International Conference on Bioinformatics and Biomedical Engineering, 2009, pp. 1-4, doi: 10.1109/ICBBE.2009.5162600.

[31] F. Abdelliche, A. Charef, and S. Ladaci, "Complex fractional and complex Morlet wavelets for QRS complex detection," ICFDA'14 International Conference on Fractional Differentiation and Its Applications 2014, 2014, pp. 1-5, doi: 10.1109/ICFDA.2014.6967456.

[32] W. Zhu, H. Zhao, and X. Chen, "A new QRS detector based on empirical mode decomposition," IEEE 10th International Conference on Signal Processing Proceedings, 2010, pp. 1-4, doi: 10.1109/ICOSP.2010.5656499.

[33] S. A. Chouakri, F. B. Reguig, and A. T. Ahmed, "QRS complex detection based on multi wavelet packet decomposition," Applied Mathematics and Computation, vol. 217, no. 23, pp. 9508-9525, Aug. 2011, doi: 10.1016/j.amc.2011.03.001.

[34] P. Phukpattaranont, "QRS detection algorithm based on the quadratic filter," Expert Systems with Applications, vol. 42, no. 11, pp. 4867-4877, Jul. 2015, doi: 10.1016/j.eswa.2015.02.012.

[35] J.-W. Lee, K. S. Kim, B. Lee, B. Lee, and M. Ho. Lee, "A Real Time QRS Detection Using Delay-Coordinate Mapping for the Microcontroller Implementation," Annals of Biomedical Engineering, vol. 30, no. 9, pp. 1140-1151, Oct. 2002.

[36] J. Lee, K. Jeong, J. Yoon and M. Lee, "A simple real-time QRS detection algorithm," Proceedings of 18th Annual International Conference of the IEEE Engineering in Medicine and Biology Society, vol. 4, 1996, pp. 1396-1398, doi: 10.1109/IEMBS.1996.647473.

[37] C. J. Deepu and Y. Lian, "A Joint QRS Detection and Data Compression Scheme for Wearable Sensors," in IEEE Transactions on Biomedical Engineering, vol. 62, no. 1, pp. 165-175, Jan. 2015, doi: 10.1109/TBME.2014.2342879.

[38] M. R. Arefin and R. Fazel-Rezai, "Computationally efficient QRS detection analysis based on dual-slope method," 2014 36th Annual International Conference of the IEEE Engineering in Medicine and Biology Society, 2014, pp. 2274-2277, doi: 10.1109/EMBC.2014.6944073.

[39] Y. Wang, C. J. Deepu and Y. Lian, "A computationally efficient QRS detection algorithm for wearable ECG sensors," 2011 Annual International Conference of the IEEE Engineering in Medicine and Biology Society, 2011, pp. 5641-5644, doi: 10.1109/IEMBS.2011.6091365.

[40] C. F. Zhang and T. Bae, "VLSI Friendly ECG QRS Complex Detector for Body Sensor Networks," in IEEE Journal on Emerging and Selected Topics in Circuits and Systems, vol. 2, no. 1, pp. 52-59, Mar. 2012, doi: 10.1109/JETCAS.2012.2187706.

[41] F. Zhang and Y. Lian, "QRS Detection Based on Morphological Filter and Energy Envelope for Applications in Body Sensor Networks," Journal of Signal Processing Systems, vol. 64, no. 2, pp. 187-194, Nov. 2009, doi: 10.1007/s11265-009-0430-8.

[42] F. Zhang and Y. Lian, "QRS Detection Based on Multiscale Mathematical Morphology for Wearable ECG Devices in Body Area Networks," in IEEE Transactions on Biomedical Circuits and Systems, vol. 3, no. 4, pp. 220-228, Aug. 2009, doi: 10.1109/TBCAS.2009.2020093.

[43] K. Arbateni and A. Bennia, "Sigmoidal radial basis function ANN for QRS complex detection," Neurocomputing, vol. 145, pp. 438-450, Dec. 2014, doi: 10.1016/j.neucom.2014.05.009.

[44] L. D. Sharma and R. K. Sunkaria, "A robust QRS detection using novel pre-processing techniques and kurtosis based enhanced efficiency," Measurement, vol. 87, pp. 194-204, Jun. 2016, doi: 10.1016/j.measurement.2016.03.015.

[45] P. Li et al., "A 410-nW Efficient QRS Processor for Mobile ECG Monitoring in 0.18- $\mu \mathrm{m}$ CMOS," in IEEE Transactions on Biomedical Circuits and Systems, vol. 11, no. 6, pp. 1356-1365, Dec. 2017, doi: 10.1109/TBCAS.2017.2731797.

[46] C.-L. Chen and C.-T. Chuang, "A QRS Detection and R Point Recognition Method for Wearable Single-Lead ECG Devices," Sensors, vol. 17, no. 9, Aug. 2017, Art. no. 1969, doi: 10.3390/s17091969.

[47] N. Ravanshad, H. Rezaee-Dehsorkh, R. Lotfi and Y. Lian, "A Level-Crossing Based QRS-Detection Algorithm for Wearable ECG Sensors," in IEEE Journal of Biomedical and Health Informatics, vol. 18, no. 1, pp. 183-192, Jan. 2014, doi: 10.1109/JBHI.2013.2274809.

[48] S. Torbey, S. G. Akl and D. P. Redfearn, "Multi-lead QRS detection using window pairs," 2012 Annual International Conference of the IEEE Engineering in Medicine and Biology Society, 2012, pp. 3143-3146, doi: 10.1109/EMBC.2012.6346631. 
[49] W. Chin, C. Chang, C. Tseng, Y. Huang and T. Jiang, "Bayesian Real-Time QRS Complex Detector for Healthcare System," in IEEE Internet of Things Journal, vol. 6, no. 3, pp. 5540-5549, 2019, doi: 10.1109/JIOT.2019.2903530.

[50] B. S. Shaik, G. V. S. S. K. R. Naganjaneyulu and A. V. Narasimhadhan, "A novel approach for QRS delineation in ECG signal based on chirplet transform," 2015 IEEE International Conference on Electronics, Computing and Communication Technologies (CONECCT), 2015, pp. 1-5, doi: 10.1109/CONECCT.2015.7383914.

[51] A. Karimipour and M. R. Homaeinezhad, "Real-time electrocardiogram P-QRS-T detection-delineation algorithm based on quality-supported analysis of characteristic templates," Computers in Biology and Medicine, vol. 52, pp. 153-165, Sep. 2014, doi: 10.1016/j.compbiomed.2014.07.002.

[52] S. Salih, S. A. Aljunid, A. Yahya, and K. Y. Ghailan, "A Novel Approach for Detecting QRS Complex of ECG signal," International Journal of Computer Science, vol. 9, no. 6, pp. 205-2015, Nov. 2012.

[53] S. Taouli and F. Bereksi-Reguig, "Detection of QRS complexes in ECG signals based on empirical mode decomposition," Global Journal of Computer Science and Technology, vol. 11, no. 20, pp. 11-17, 2011.

[54] Y. Yeh and W. Wang, "QRS complexes detection for ECG signal: The Difference Operation Method," Computer Methods and Programs in Biomedicine, vol. 91, no. 3, pp. 245-254, Sep. 2008, doi: 10.1016/j.cmpb.2008.04.006.

[55] M. Paoletti and C. Marchesi, "Discovering dangerous patterns in long-term ambulatory ECG recordings using a fast QRS detection algorithm and explorative data analysis," Computer Methods and Programs in Biomedicine, vol. 82, no. 1, pp. 20-30, Apr. 2006, doi: 10.1016/j.cmpb.2006.01.005.

[56] J J. Pan and W. J. Tompkins, "A Real-Time QRS Detection Algorithm," in IEEE Transactions on Biomedical Engineering, vol. BME-32, no. 3, pp. 230-236, Mar. 1985, doi: 10.1109/TBME.1985.325532.

[57] Z.-E. Hadj Slimane and A. Naït-Ali, "QRS complex detection using Empirical Mode Decomposition," Digital Signal Processing, vol. 20, no. 4, pp. 1221-1228, Jul. 2010, doi: 10.1016/j.dsp.2009.10.017.

[58] P. S. Hamilton and W. J. Tompkins, "Quantitative Investigation of QRS Detection Rules Using the MIT/BIH Arrhythmia Database," in IEEE Transactions on Biomedical Engineering, vol. BME-33, no. 12, pp. 1157-1165, Dec. 1986, doi: 10.1109/TBME.1986.325695.

[59] C. Ieong, P. Mak, M. Vai and R. P. Martins, "Sub- $\mu$ W QRS detection processor using quadratic spline wavelet transform and maxima modulus pair recognition for power-efficient wireless arrhythmia monitoring," 201621 st Asia and South Pacific Design Automation Conference (ASP-DAC), 2016, pp. 21-22, doi: 10.1109/ASPDAC.2016.7427982.

[60] J. P. Sahoo et al., "Autocorrelation and Hilbert transform-based QRS complex detection in ECG signal," International Journal of Signal and Imaging Systems Engineering, vol. 7, no. 1, 2014, Art. no. 52, doi: 10.1504/IJSISE.2014.057939.

[61] Z. Zhang et al., "A Kalman filtering based adaptive threshold algorithm for QRS complex detection," Biomedical Signal Processing and Control, vol. 58, Apr. 2020, Art. no. 101827, doi: 10.1016/j.bspc.2019.101827.

[62] X. Lu et al., "QRS Detection Based on Improved Adaptive Threshold," Journal of Healthcare Engineering, vol. 2018, pp. 1-8, 2018, doi: 10.1155/2018/5694595.

[63] X. Zhang and Y. Lian, "A 300-mV 220-nW Event-Driven ADC With Real-Time QRS Detection for Wearable ECG Sensors," in IEEE Transactions on Biomedical Circuits and Systems, vol. 8, no. 6, pp. 834-843, Dec. 2014, doi: 10.1109/TBCAS.2013.2296942.

[64] S. Farashi, "A multiresolution time-dependent entropy method for QRS complex detection," Biomedical Signal Processing and Control, vol. 24, pp. 63-71, Feb. 2016, doi: 10.1016/j.bspc.2015.09.008.

[65] A. Chen et al., "A Real Time QRS Detection Algorithm Based on ET and PD Controlled Threshold Strategy," Sensors, vol. 20, no. 14, Jul. 2020, Art. no. 4003, doi: 10.3390/s20144003.

[66] X. Tang, Q. Hu, and W. Tang, "A Real-Time QRS Detection System With PR/RT Interval and ST Segment Measurements for Wearable ECG Sensors Using Parallel Delta Modulators," in IEEE Transactions on Biomedical Circuits and Systems, vol. 12, no. 4, pp. 751-761, Aug. 2018, doi: 10.1109/TBCAS.2018.2823275.

[67] Y. Zhao, Z. Shang, and Y. Lian, "User Adaptive QRS Detection Based on One Target Clustering and Correlation Coefficient," 2018 IEEE Biomedical Circuits and Systems Conference (BioCAS), 2018, pp. 1-4, doi: 10.1109/BIOCAS.2018.8584803.

[68] M. Elgendi, A. Mohamed, and R. Ward, "Efficient ECG Compression and QRS Detection for E-Health Applications," Scientific Reports, vol. 7, no. 1, Mar. 2017, doi: 10.1038/s41598-017-00540-x.

[69] J. Darrington, "Towards real time QRS detection: A fast method using minimal pre-processing," Biomedical Signal Processing and Control, vol. 1, no. 2, pp. 169-176, Apr. 2006, doi: 10.1016/j.bspc.2006.08.002.

[70] A. Ghaffari, H. Goldbayani, and M. Ghasemi, "A new mathematical based QRS detector using continuous wavelet transform," Computers \& Electrical Engineering, vol. 34, no. 2, pp. 81-91, Mar. 2008, doi: 10.1016/j.compeleceng.2007.10.005.

[71] Z. Hou, Y. Dong, J. Xiang, X. Li, and B. Yang, "A Real-Time QRS Detection Method Based on Phase Portraits and Box-Scoring Calculation," in IEEE Sensors Journal, vol. 18, no. 9, pp. 3694-3702, May 2018, doi: 10.1109/JSEN.2018.2812792.

[72] J. Rahul, M. Sora, and L. D. Sharma, "Exploratory data analysis based efficient QRS-complex detection technique with minimal computational load," Physical and Engineering Sciences in Medicine, vol. 43, no. 3, pp. 1049-1067, Jul. 2020, doi: 10.1007/s13246-020-00906-y. 


\section{BIOGRAPHIES OF AUTHORS}
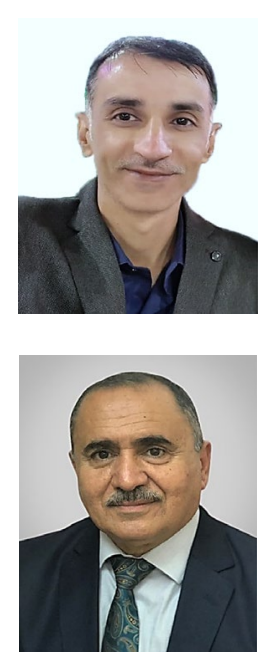

Akram Jaddoa Khalaf was born in Hilla, Babylon, Iraq, in 1978. He received the B.Sc. degree in Electrical Engineering in 2001 from the University of Babylon, the HD degree in Computer Science/Engineering Applications in 2002 from the Iraqi Commission for Computer \& Informatics, and the M.Sc. degree in Electrical Engineering/Electronics and Communications in 2013 from the University of Babylon. He is a staff member at the University of Babylon, Faculty of Engineering, Biomedical Department, and he is currently a Ph.D. student at the University of Babylon. His research interests include Wireless Sensor Networks, Biomedical informatics, Signal Processing, Healthcare systems, Networking, and Machine Learning.

Samir Jasim Mohammed was born in Hilla, Babylon, Iraq, in 9 Oct. 1959. He received the B.Sc. degree in Electrical Engineering/Electronics and Communications in 1981 from the University of Sulaymaniyah, the M.Sc. degree in Electrical Engineering/Electronics and Communications in 1986 from the University of Baghdad, and the Ph.D. degree in Communication Engineering from the Department of Technical Education-Electrical Engineering at University of Technology. Currently, he is professor and Head of the Scientific Composition at the Electrical Engineering Department, University of Babylon. His research interest is Wireless Communication, Digital Video Broadcasting, Wireless sensor network, Biomedical informatics, Signal Processing, Healthcare System. 